\title{
IS SUPERFICIAL KNOWLEDGE ABOUT STELLAR SURFACES SUFFICIENT?
}

\author{
BENGTGUSTAFSSON \\ Astronomiska Observatoriet, Box 515, \\ S-751 20 Uppsala, Sweden
}

\begin{abstract}
The significance of a detailed knowledge of the atmosphere of a star for a proper understanding of its interior is illustrated and discussed. It is concluded that significant progress in the understanding of stellar atmospheres is needed in order to match methods, discoveries and needs in the study of stellar internal structure and evolution.
\end{abstract}

\section{INTRODUCTION}

In his classical textbook, Stucture and Evolution of the Stars, Martin Schwarzschild (1958) discussed the question of the outer boundary conditions (at $r=R$ ). The author found that, although the zero condition ( $T=0$, $P=0$ ) was adequate for stars with radiative envelopes (of spectral types earlier than, say, A5), for later spectral types it was not satisfactory due to convection. In that case he defined the "convective zero solution", the straight extrapolation of the simple adiabatic relation all the way out to the surface. He then argued that the exact solution only differs appreciably at temperatures lower than fifty thousand degrees and, "since errors restricted to such very low temperatures will have virtually no consequences for our results for the stellar interior, we may use the simple adiabatic relation throughout the convection zone to the very edge." If this were true my task here, having been asked to review "the role of model atmospheres for understanding stellar structure" would have been simply reduced to that of the interested listener. However, things are, 34 years later, not so easy any longer.

In 1958 only few models of stellar atmospheres that considered the 
effects of the wavelength dependence of the opacity sources (so-called "nongray models") had been calculated. Now, very extensive grids of such models, with consideration of a number of continuos opacity sources and of the blanketing of millions of spectral lines, are available for late-type stars. In spite of this sophistication, these models are still quite primitive in the sense that they are static and stratified in plane-parallel layers. Convective energy transport is generally modelled with the mixing-length theory (MLT), and at least in most cases LTE is assumed to prevail. The question has been much debated to which extent this "speculative-classical theory" (Thomas, 1989) is useful at the interpretation of observational data like stellar spectra. We shall see that its use may be even more questionable when boundary conditions for contemporary studies of stellar interiors are needed.

Models of stellar atmospheres are needed for a number of different purposes when one wants to study the interior of stars. First of all, the understanding of diagnostics for effective temperature, gravity, and atmospheric chemical composition is not only vital when putting the stars onto the $\log L-T_{\text {eff }}$ diagram for comparison with stellar models but also when tracing the results of evolutionary processes in the dredge up, burning or settling of various chemical elements. That is illustrated in Poul Erik Nissen's contribution to the present proceedings and I shall only add a few comments on this important aspect of application of model atmospheres. Instead, I shall dwell on more physical connections between the outer and the inner regions of stars, such as the determination of radius (or estimation of mixing length) and the significance of atmospheric opacities, and the relation between the atmospheric processes and stellar oscillations, magnetic fields, angular momentum and internal mixing processes. I shall essentially confine my discussion to solar-type stars, in spite of the temptation to glance at stars in other parts of the HR diagram to find more drastic examples to illustrate my leading theses. Obviously, my discussion will be very far from comphrehensive; any reader who would wish a more systematic presentation of stellar atmospheres and related problems for solar-type stars is advised to consult the excellent monograph by Cram and Kuhi (1989). Also, many of the contributions at this conference will contain additional details and further examples along the lines of my presentation - such as the reviews by Ulrich, Zahn, Michaud and Proffitt, Christensen-Dalsgaard, Krause, Schriver and MacGregor, and several others. 


\section{IHE DETERMINATION OF FUNDAMENTAL PARAMETERS}

The art of deriving stellar fundamental parameters from stellar spectra, using model atmospheres, has been repeatedly summarized in recent years (see, e.g., Gustafsson and Jørgensen (1985), Spite and Spite (1985), Gehren (1988), Lambert (1989), and Wheeler, Sneden and Truran (1989)). Also, a great number of impressive results have been obtained, as was also illustrated in the Nissen's presentation. Here, only a few aspects will be discussed, of particular significance for solar-type stars.

It was since long recognized that the ultraviolet flux of solar-type stars was not properly reproduced by classical model atmospheres. Even if all known spectral lines of atoms and molecules were taken into account the theoretical fluxes were too strong, in the solar case by typically $10 \%$, and in cooler stars by much more (cf. Gustafsson et al. 1975, Gustafsson and Bell 1979). Following Holweger (1970) and others we ascribed this failure to the presence of a "veil" of many very weak metal lines in the late-type stellar spectra. Recently, Bob Kurucz has completed his calculation of a very detailed line list with more than $2 \times 10^{7}$ metal lines, giving identifications, wavelengths, excitation energies, gf-values, damping constants and Landéfactors. He has also shown that when this line list is used a good agreement is obtained between observed and calculated fluxes of the Sun in the near ultraviolet (Kurucz 1991). We have found similar results, using Kurucz's list but otherwise a completely different computer code. It could be remarked that, when scrutinizing synthetic solar spectra calculated with Kurucz's data for the metal lines in the $3300 \dot{A}$ region we have found very great discrepancies (typically a factor of 10 ) between calculated and observed metal line strengths. It seems, however, that statistically the veil of weak metal lines is very vell described. We have also found a resonably good agreement between calculated and observed uv fluxes for $F$ stars of different metallicities (cf. Edvardsson et al. 1992).

When introducing the new ultraviolet opacity data we also found a gratifying agreement between surface gravities for $F$ stars, normally deduced from photometric criteria calibrated basically on parallaxes, and those one would derive from ionization equilibria. This is illustrated in Fig. 1, where the iron abundances deduced from $\mathrm{Fe} I$ and $\mathrm{Fe} I \mathrm{I}$ lines, respectively, are plotted relatively to each other for our sample of solar-type disk stars with different metal abundances. The agreement is much better than one could 
derive from our previous set of models and not dependent on the stellar temperature or metallicity. Likewise, the abundances deduced from lines of different excitation agree very well. These findings and a number of other results suggest that the effects of overionisation due to the non-local radiative field, or of temperature inhomogeneities, are not very severe for abundance analysis of solar-type disk stars, as long as a strictly differential approach is taken relative to the Sun and weak spectral lines are used. One may still suspect, however, that such problems may plague, e.g., detailed analyses of Pop. II dwarts.

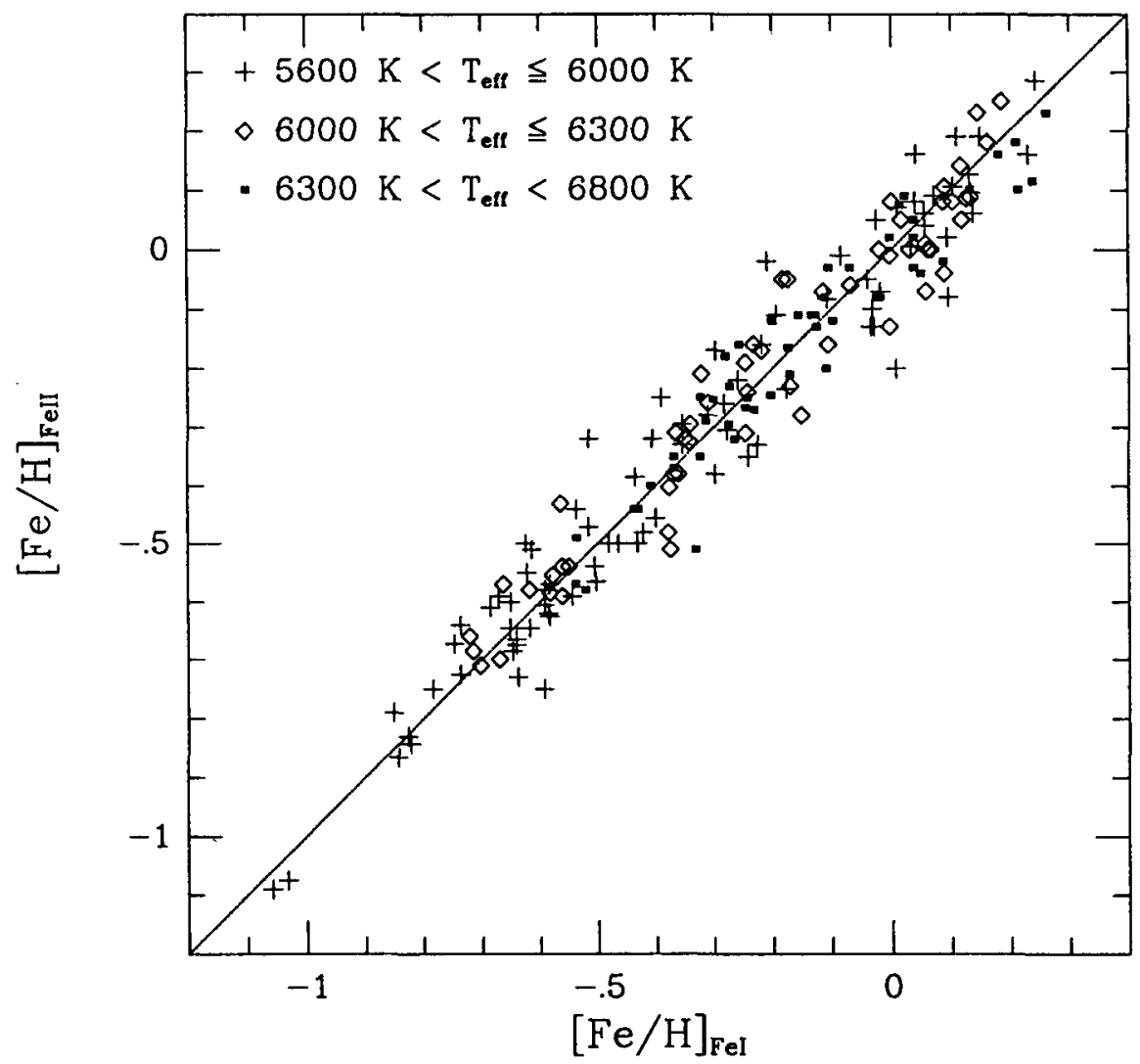

Fig. 1. Iron abundances derived for almost 200 solar-type disk dwarfs from $\mathrm{Fe} I$ and $\mathrm{Fe} I \mathrm{l}$ lines, respectively. The symbol for each star is depending on the stellar effective temperature. The line is a one-to one line. Obviously, there is no significant difference, depending on stellar temperature or metallicity, in the abundances from neutral or ionized Fe lines. (From Edvardsson et al. 1992.) 
An interesting result of our extensive abundance survey for disk dwarfs (Edvardsson et al. 1992) is the location of the Sun in the abundance space. We thus find that the solar abundances of, e.g., $\mathrm{Mg}$ and Si, seem low by typically $25 \%$, while the $\mathrm{Ca}$ abundance seems normal and $\mathrm{O}$ is high, with respect to $\mathrm{Fe}$. Is this effect really real? We do not think it can be due to errors in the estimated colours of the Sun (i.e. in the zero-point in the temperature scale), nor in the gf values, which were estimated from the solar equivalvent widhts, measured in the same way as those of the stars. Nor should it be the result of errors in the model atmospheres, if the solar atmosphere is not systematically different from those of stars with similar effective temperatures, gravities and iron abundances. We argue that the reason probably is that the Sun is somewhat poor by typically -0.1 dex in many chemical elements, relative to iron, as compared with the mean abundances of similar stars in the solar neigbourhood. This may be of significance when one relies on our star as a calibration standard.

Presumably these examples, as well as Nissen's contribution to these proceedings, show that rather interesting results may be obtained from a careful use of classical models to calibrate spectroscopic observations of stellar photospheres. Yet, one should note that the classical and static models were designed mainly to be used for predicting photospheric radiation fluxes, while they will fail totally in describing most dynamic phenomena.

\section{MIXINGLENGTH AND OPACITIES}

For some time model atmospheres have been used as outer boundary conditions for models of stars with extensive convection zones. In fact, this is connected with estimating the mixing length parameter $\alpha=2 / \mathrm{H}$, with 2 being the mixing length and $H$ the scale hight. If $\boldsymbol{Z}$ is fixed by using the Sun with its well known radius, age and mass one can then use the temperature-pressure scales of a suitable grid of model atmospheres as boundary conditions, assuming $\alpha$ to remain the same.

One example of this is the extensive grid calculated by VandenBerg (1983) where the models by Bell et al. (1976 and later) were used for establishing the "photospheric pressures", i.e. the gas pressures at the depth $\tau_{\text {eff }}$ where $T=T_{\text {eff }}$. (In principle, one should sooner use a boundary point deeper into the atmosphere where the optical depth at the most transparent wavlength is $\gg 1$, but the choice of $\tau_{\text {eff }}$ was indeed found to be satisfactory, at 
least for $T_{\text {eff }}$ around $5000 \mathrm{~K}$, by Pedersen, VandenBerg and Irwin (1990.) However, VandenBerg and Laskarides (1987) preferred to revert back to the more primitive boundary conditions for the following reason: If model atmospheres were used, the properties of red giants were well discribed by the models over a wide range in metallicity with the value of the mixinglength parameter $\alpha=1.5$. However, for the Sun this grid of models with $\alpha=$ 1.5 gave a predicted $T_{\text {eff }}$ for a solar model which was significantly too hot, by more than $100 \mathrm{~K}$. Instead of fixing this by lowering the solar value of $\alpha$ VandenBerg and Laskarides argued that this failure might be due to the missing ultraviolet opacity in the model atmospheres. This conjecture has been checked with the new model atmospheres (Edvardsson et al. 1992) as boundary conditions. Indeed, there is a very substantial improvement for the Sun. As is seen in Fig. 2 the solar model now falls almost exactly in the right position in the theoretical HR diagram.

In spite of this success one should give a strong warning concerning these plane-parallel model atmospheres, with mixing-length convection. The atmospheres of true stars in this parameter range, like that of the Sun, are known to be strongly structured by non-local convection and radiation cooling in intricate interplay, leading to considerable inhomogeneities in temperature and velocity fields (granulation). The detailed numerical 3D simulations by Nordlund and Dravins (1990) clearly showed the inadequacy of standard models, even for determining the mean temperature structure of these photospheres. What the traditional mixing-length theory succeeds in describing is the entropy jump accross the shallow super-adiabatic layer near the stellar surface. Rather different local convection theories with free parameters may be calibrated fit the global solar parameters (cf. Gough and Weiss, 1976) but there is no guarantee that this parameter will be the same for different stars. Nordlund and Dravins demonstrated that the standard MLT with a reasonably constant value of $\alpha$ (close to 1.5) can be used at least within a parameter range characteristic of solar-type stars (see, however, Steffen's contribution to the present proceedings). For red giant stars, it still remains to be investigated whether this is true.

No doubt, the considerable improvements that have taken place in recent years concerning atomic opacities affect the stellar interior models considerably. This is further commented on by $M$. Seaton in the present proceedings, and I shall therefore limit my comments to a few words about molecular opacities (for a more general review, see Jorgensen 1992). The effects of molecules are mainly limited to the cool end of the HR diagram, and 


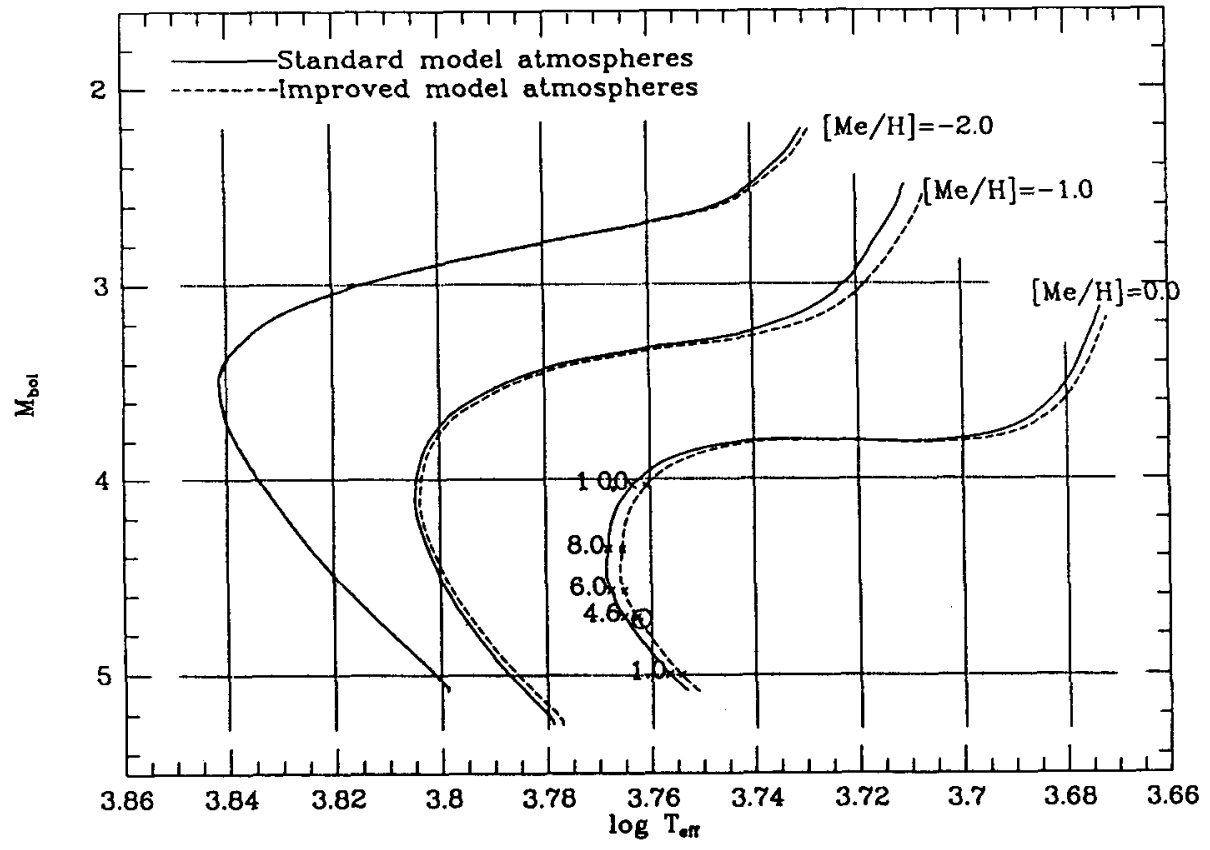

Fig. 2 Evolutionary tracks for $\alpha=1.5$, calculated by D. A. VandenBerg (1992) using model atmospheres from the grid of Bell et al. (1975, full lines) and a grid with improved uv blanketing (cf. Edvardsson et al. 1992). Time in Gyears and the solar position is shown at the $[\mathrm{Me} / \mathrm{H}]=0.0$ tracks. The tracks are still preliminary; the opacities can be further improved. Note the different effects of the new boundary conditions for different metallicities.

in particular to the red giants, whose diameters are very considerably affected by the molecular opacities. (We found an extreme effect of this kind some years ago when introducing polyatomic molecular opacities into model atmospheres for carbon stars (Eriksson et al. 1984). The introduction of HCN for $T_{\text {eff }}$ around $3000 \mathrm{~K}$ expanded the model atmosphere such that the radius of the star could grow by a factor of two or more, as compared with a model without any polyatomics.) For interior models of $A B G$ stars Sackman and Boothroyd (1991), using the new LAOL opacity tables which included opacities from $\mathrm{CO}, \mathrm{CN}$ and $\mathrm{H}_{2} \mathrm{O}$, also found very significant effects on the radii. In order to restore agreement with observed giant branches it was found necessary to increase $\alpha$ on the AGB to more than 2.0. For further studies of these effects it is obviously important that reasonably realistic model atmospheres, including all relevant molecular opacities and sphericity 
effects, are used for setting the boundary conditions. As soon as possible, dynamic models with more realistic representation of convection similar to contemporary simulations for the solar type stars should be attempted.

For models of stars of about solar temperatures the effects of the molecular opacities are presumably so limited that the errors in them should not lead to very significant model errors, in spite of the fact that also for diatomic molecules the present situation as regards absorption coefficients is far from satisfactory (cf. Jørgensen 1992).

\section{ATMOSPHERES AND DYNAMIC PROCESSES INTHE INTERIOR}

\section{a. Stellar oscillations}

Although the effect of the outer layers on the interior of static stellar models is often negligible the corresponding is not necessarily true when dynamic processes are taken into account. For solar-type stars the question of the driving source of the solar "five-minute" oscillations has been debated intensively. Although these oscillations probably do not affect the grand structure of the interior layers, they are very significant diagnostics of this structure. It is therefore important to understand their physical origin, not the least when judging the possibilities of observing corresponding phenomena for other stars (see Christensen-Dalsgaard and Frandsen 1983).

The power spectrum of the solar oscillations is practically independent of the degree $(\ell)$ of the modes for $\ell<100$ (Christensen-Dalsgaard and Gough 1982, Libbrecht et al. 1986). This means that the oscillation amplitudes are not dependent on the horizontal wavenumbers, suggesting that the amplitudes are determined in layers where the acoustic waves propagate vertically, i.e. close to the surface. Several recent studies have stressed the probably very significant role that the upper boundary of the convection zone plays for exciting and damping the oscillations. In a series of theoretical papers Balmforth (1992a-c) has investigated the physical character of solar oscillations - their thermodynamics, frequencies and amplitudes. Although the question whether the solar pulsation modes are self-excited or stochastically excited by turbulent convection is not totally solved, Balmforth's study gives most support to the latter mechanism. He also finds a good agreement between calculated and observed damping rates, except for waves of high frequencies which propagate out into the atmosphere before being reflected back into the interior. Balmforth suggests that that mismatch 
is because the mixing-length theory does not take horizontal inhomogeneities into consideration. High-order oscillation modes, with wavelengths comparable to the scales of these inhomogeneities, should experience incoherent scattering by these inhomogeneities in the superficial layers.

Recently extensive numerical simulations have been carried out for the Solar atmosphere by Nordlund and Stein (1991a and b) of convection and waves with a method that allows compression and shocks. They find that the switch from convective to radiative energy transport at the solar surface causes non-adiabatic fluctuations in the gas pressure that stochastically excite the p-modes. In fact, both the excitation and the linear damping mechanisms of these waves are concentrated near the surface.

The detailed structure of the surface layers, which is only partially known mainly because of the unsatisfactory theory of convection, also effects the frequencies. Balmforth (1992b) has investigated the small but significant errors on the 0.5 percent level between calculated and observed frequencies for the $5 \mathrm{~min}$ oscillations. He suggests that the discrepancy is due to the modelling of the upper solar boundary layers with the conventional mixing-length theory. Indeed, the recent simulations by Stein and Nordlund (1991b) give such effects of the right order of magnitude due to convective overshoot and turbulent pressure support. Also at the calculation of amplitudes the details of the upper layers play a decisive role (cf. Christensen-Dalsgaard and Frandsen, 1983, Balmforth 1992c).

We thus find that the shortcomings of the understanding of the upper stellar layers have direct consequences for the interpretation of solar, and stellar, oscillations. When once measured, the stellar oscillation power spectra should contain new information on the stellar surface gravity and effective temperature. It is, however, not clear to me how useful this information may be, in view of the uncertainties discussed above. In their recent review Gough and Toomre (1991) conclude: "The greatest helioseismological uncertainties lie in the superficial layers of the Sun." On the other hand this means that observations of oscillations, when combined with reasonably sound theoretical models, will give further valuable information as regards the structure of the surface layers.

\section{b. Magnetic fields, mass loss, and momentum transport}

The relation between convection, rotation, magnetic field, mass loss and mixing in stars is complex and interesting. One could ask to which extent the structure of the outer layers of the star play an active role in this interplay. 
In outlining this we shall start with the magnetic fields and convection, and then proceed to the angular momentum transfer and mixing processes.

Magnetic fields may connect the structure and evolution of the interior of a star to its boundary in intricate ways. Recently, Charbonneau and MacGregor (1992a) have simulated the angular momentum transport in stellar magnetic and, initially, nonrotating radiative zones, around a spinning core. They find a rich variety of behaviour: time scales and evolution are critically dependent on the way the magnetic field is anchored in the core and its behaviour at the surface (i.e. the bottom of the convective envelope).

The convection simulations of Stein and Nordlund (1989) and Nordlund and Stein (1991a) led to the interesting dicovery that the topology of solar convection at great atmospheric depths is qualitatively very different from that closer to the surface. The subsurface structure is characterized by thin filaments of cool gas, downdrafts decending from the surface in a background of slowly ascending, very nearly isentropic gas. Moreover, the cold filaments seem to merge into larger and more widely separated filaments at successively larger depths. This structure of downdrafts, containing magnetic flux-tubes, may be of great significance for the way the magnetic fields are generated by the dynamo in the lower boundary layer of the convection zone. Still, one may speculate that this structure could be critically dependent on the conditions in the atmosphere where the radiative cooling of the hot rising gas takes place. We note that Cattaneo et al. (1991) find similar structures in their $3 \mathrm{D}$ simulations of compressible convection, but that it is not clear yet how sensitive these structures are to the boundary conditions.

Interesting simulations of dynamos in 3D convection models of this type have recently been produced by Nordlund et al. (1992). In these simulations, spontaneous dynamo action occurs at the lower boundary of the convection zone, with most of the magnetic field being generated as coherent flux tubes in the vincinity of strong downdrafts. The mechanism is closely related to the "stretch-twist-fold" dynamo, suggested by Vainshtein and Zeldovich (1972).

Although the applicability of the results discussed above is very uncertain they point at the possibility that the stellar boundary region, $i$. e. the atmosphere and convective envelope, and its structure, may be of great significance in affecting the evolution of magnetic fields and angular momentum in the interior.

The wellknown observations that the mean rotation speed of main sequence (MS) stars drops drastically at spectral types around $F 0$ is generally ascribed to the fact that stars with masses below about 1.5 solar 
masses have relatively deep convective envelopes. These convective zones are thought to promote dynamo action and magnetic braking. The latter is a consequence of the torque applied to the star by its magnetically coupled wind, as was first suggested by Schatzman (1962). This magnetic braking in the pre-MS or early MS life of the stars is probably a key significance in determining the stellar rotation pattern.

The theory of angular momentum loss from the star due to magnetic braking was summarized by Mestel (1984) and further developed by Kawaler (1988). The angular momentum loss rate depends on the mass loss rate $\dot{M}$, the surface magnetic field strength and the magentic field configuration in the upper atmosphere. There is still no convincing theory for winds from solartype stellar atmospheres (including the pre-MS stage) that would allow us to successfully predict $\dot{M}$ from more basic quantities, such as fundamental parameters of the photosphere and a measure of the mechanical energy flux through it. It is not even known whether such a theory is physically correct at all, or whether $\dot{M}$ should also be added to the list of fundamental atmospheric parameters (cf. the discussion referred to by Cram 1989 and references cited therein, Thomas 1989, Cannon and Thomas 1977). However, in the calculations of the transfer of angular momentum in the Sun Pinsonneault et al. (1989) find a rather small effect of "moderate" changes in $\dot{M}$ on the resulting distribution of angular momentum. These authors show, on the other hand, that the magnetic field strength and other features of the wind model have a dominant influence on the surface rotation for a mature main-sequence star, like the Sun, as well as for rotationally induced mixing. Clearly, considerable progress has to be made in the modelling of stellar magnetic fields and winds before the magnetic braking can be predicted with certainty.

The solar model evolved by Pinsonneault et al. included angular momentum redistribution by rotationally induced hydrodynamical instabilities of various kinds and at various scales. Some free parameters were set such that a good agreement with solar data was obtained. A rapidly rotating central core persisted. Zahn (1992) has recently developed a more detailed analytical treatment of the meridional circulation and turbulence in a rotating model, assuming turbulence to be anisotropic with a greater horizontal than vertical transport. He predicts a rapidly spinning solar core, concludes that the slowly rotating old Pop. II dwarfs all should have more or less depleted $\mathrm{Li}$ abundances and argues for the "wind-driven circulation" as the relevant concept - the wind brakes and that causes the circulation.

As an alternative to the transfer of angular momentum through the stars 
by hydrodynamical means mediation by internal magnetic fields has been proposed (e.g., by Mestel and Weiss 1987). Recently Charbonneau and MacGregor (1992a and b) have studied this further on the basis of numerical simulations and obtained significantly lower core rotation velocities due to the magnetic copuling between the core and the layers outside it. They demonstrate the significane of the internal magnetic field configuration for the rotational behaviour of solar-type stars, but also find that the present solar surface rotation rate is a very poor indicator of the strength and geometry of the internal fields. The surface rotation in the early ages, or the present internal differential rotation, are much more dependent on these fields.

\section{c. Diffusion and settling}

Another example of processes where the atmosphere and its structure affects the interior are the diffusion processes. While, for early-type stars, helium settles gravitationally, the heavier elements either diffuse inwards or outwards, depending on whether the outward radiative acceleration is greater or smaller than the acceleration of gravity (cf., e.g., Michaud 1991, and references cited therein). This leads to interesting consequences for the internal structure - e.g. that the $\mathrm{He}$ II convection zone vanishes when diffusion proceeds and He gets strongly depleted. The atmosphere plays a significant role, setting the boundary conditions for this process. Significant atmospheric properties that may determine what happens are mass loss and the evolution of surface magnetic fields.

Even if similar phenomena may occur at greater depths in cooler stars, the importance of the atmosphere for these processes is probably much less, since it is situated on the top of the deep hydrogen convection zone. We note, however, that Michaud (1991) seems to find significant settling effects on surface abundances for main sequence stars with $T_{\text {eff }}$ as low as $6000 \mathrm{~K}$.

\section{CONGLUSIONS}

I hope that this presentation has demonstrated that the interrelation between stellar interiors and atmospheres is far more intricate than the atmosphere just being a single-valued boundary condition. It was early appreciated that static model atmospheres are not very helpful for the analysis of criteria showing the presence of stellar chromospheres, coronae and winds. Likewise, if the range of new methods for exploring stellar structure and evolution are to 
be exploited, the description of the atmosphere as a static and inactive planeparallel zone on top of the star is insufficient. One way to circumvent this problem is to develop parametrized "theories" where the parameters are determined from observations. Examples of simple such approaches are the mixing-length theory or the modelling of magnetic braking discussed above. For many tasks these recipes may be acceptable, although one will have to wait for a detailed physical theory before this can be proven. The free parameters, however, may not have a sound physical interpretation, and there is no guarantee that they stay constant for a given star or equal for different but similar stars. Basically worse is that it may not lead to a correct understanding of the underlying physics, not even to the formulation of new relevant hypotheses. The physical connections between the atmosphere and the interior are dynamical and complex phenomena and need systematic studies, aiming at detailed semi-empirical modelling of the dynamic behaviour of a number of stars. Reasonably realistic numerical simulations, compared with high-resolution (spectral and if possible spatial) monitoring will continue to play the key role here. Fortunately, the methodological development in these areas is presently very promising. Hopefully, in some years we shall know much more, also about how far from complex realities our ideas are.

\section{ACKNOWLDEEMENT}

Thanks are due to D. A. VandenBerg for calculating the evolutionary tracks shown in Fig. 2.

\section{REFERENCES}

Balmforth, N. J. 1992a, b, c, M.N.A.A.S. 255, 603, 632, 639.

Bell, R. A., Eriksson, K., Gustatsson, B., Nordlund, A. 1976, A.\&Ap. Suppl. $23,37$. Cannon, C. J., Thomas, R. N. 1977, Ap.J. 211, 910.

Cattaneo, F., Brummell, N. H., Toomre, J., Malagoli, A., Hurlburt, N. E. 1991, Ap.J. 370, 282.

Charbonneau, R., MacGregor, K. B. 1992a, Ap.J. 387, 639.

Charbonneau, R., MacGregor, K. B. 1992b, Ap.J. Letters, submitted.

Christensen-Dalsgaard, J., Gough, D. O. 1982, M.N.R.A.S. 198, 141.

Christensen-Dalsgaard, J., Frandsen, S. 1983, Solar. Phys. 82, 165.

Cram, L. E. 1989, in Cram and Kuhi 1989, p. 181.

Cram, L. E., Kuhi, L. V. 1989 (eds.), FKG stars and T Tauri Stars, Monograph Series on Nonthermal Phenomena in Stellar Atmospheres, NASA SP-502. 
Edvardsson, B., Andersen, J., Gustatsson, B., Lambert, D. L., Nissen, P. E., Tomkin, J. 1992, A.\&Ap.,to be submitted.

Eriksson, K., Gustafsson, B., Jørgensen, U. G., Nordlund, À. 1986, A.\&Ap.132, 37. Gehren, T. 1988, in Rev. Mod. Astron. 1, 52, G. Klare (ed.) (Springer: Heidelberg). Gough, D. O., Weiss, N. O. 1976, M.N.R.A.S. 176, 589.

Gough, D., Toomre, J. 1991, Ann. Rev. A. Ap. $29,627$.

Gustafsson, B., Bell, R. A., Eriksson, K., Nordlund, A. 1975, A.\&Ap. 42, 407.

Gustafsson, B., Jørgensen, U. G. 1985, in Proc. IAU Symp. 111: Calibration of Fundamental Stellar Quantities, D. S. Hayes, L. E. Pasinetti, A. G. D. Philip (eds.) (Reidel: Dordrecht) p. 303.

Holweger, H. 1970, A.\&Ap. 4, 11.

Jørgensen, U.G. 1992, Rev. Mex. A. Ap., in press.

Kawaler, S. D. 1988, Ap.J. 333, 236.

Kurucz, R. 1991, in Stellar Atmospheres: Beyond Classical Models, L. Crivellari, I. Hubeny, D. G. Hummer (eds.), NATO ASI-Series C 341.

Lambert, D. L. 1989, in Cosmic Abundances of Matter, C. Jake Waddington (ed.), AlP Conf. Proc. 183, 168.

Libbrecht, K.G., Popp, B.D., Kaufman, J.M., Penn, M.J. 1986, Nature 323, 235.

Mestel, L. 1984, in 3rd Cambridge Workshop on Cool Stars, Stellar Systems and the Sun,S. L. Baliunas, L. Hartmann (eds.) (Springer: New York), p. 49.

Mestel, L., Weiss, N. O. 1987, M.N.R.A.S. 226, 123.

Michaud, G. 1991, in Proc. IAU Symp. 145, Evolution of Stars: The Photospheric

Abundance Connection, G. Michaud, A. Tutukov (eds.) (Kluwer Acad.: Dordrecht), p. 111.

Nordlund, A., Dravins, D. 1990, A.\&Ap. 228, 155.

Nordlund, A., Stein, R. F. 1991a, in Stellar Atmospheres: Beyond Classical Models, L. Crivellari, I. Hubeny, D. G. Hummer (eds.), NATO ASI-Series C 341, 263.

Nordlund, A., Stein, R. F. 1991b, Proc. of the Mini Workshop on the Solar 5 Minutes Oscillations, Univ. of Oslo.

Nordlund, Ȧ., Brandenburg, A., Jennings, R. J., Rieutord, M., Ruokolainen, J., Stein, R. F., Tuominen, I. 1992, Ap.J. 392, 647.

Pedersen, B. B., VandenBerg, D. A., Irwin, A. W. 1990, Ap.J. 352, 279.

Pinsonneault, M. H., Kawaler, S. D., Sofia, S., Demarque, P. 1989, Ap.J. 338, 424.

Sackmann, I.-J., Boothroyd, A. I. 1991, Ap.J. 366, 529.

Schatzman, E. 1962, Ann. d'Astrophys. 25, 18.

Schwarzschild, M. 1958, Structure and Evolution of the Stars,(Princeton Univ. Press).

Spite, M., Spite, F. 1985, Ann. Rev. A. Ap. 23, 225.

Stoin, R. F., Nordlund, Á. 1989, Ap.J. Letters 342, L95.

Stein, R. F., Nordlund, A. 1991, in Challenges to Theories of the Structure of Moderate Mass Stars, J. Toomre and D. O. Gough (eds.), Lecture Notes in Physics 388, 195 (Springer: Heidelberg).

Thomas, R. N. 1989, in Cram and Kuhi (1989), p. ix.

Vainshtein, S. I., Zeldovich, Ya., B. 1972, Sov. Phys. Usp. 15, 159.

VandenBerg, D. A. 1983, Ap.J. Suppl. 51, 29.

VandenBerg, D. A. 1992, private communication.

VandenBerg, D. A., Laskarides, P. G. 1987, Ap.J. Suppl. 64, 103.

Wheeler, J. C., Sneden, C., Truran Jr., J. W. 1989, Ann. Rev. A. Ap. 27, 279.

Zahn, J.-P. 1992, A.\&Ap., submitted. 